\title{
Comparing the antidiabetic effects and chemical profiles of raw and fermented Chinese Ge-Gen-Qin-Lian decoction by integrating untargeted metabolomics and targeted analysis
}

Yan Yan ${ }^{1+}$, Chenhui Du ${ }^{2+}$, Zhenyu Li $i^{1}$, Min Zhang ${ }^{1,3}$, Jin Li ${ }^{2}$, Jinping Jia ${ }^{1}$, Aiping Li ${ }^{1}$, Xuemei Qin ${ }^{1 *}$ and Qiang Song2 ${ }^{2 *}$ (1)

\begin{abstract}
Background: Microbial fermentation has been widely applied in traditional Chinese medicine (TCM) for thousands of years in China. Various beneficial effects of fermentation for applications in TCM or herbals have been reported, such as enhanced anti-ovarian cancer, antioxidative activity, and neuroprotective effects. Ge-Gen-Qin-Lian decoction (GQD), a classic TCM formula, has been used to treat type 2 diabetes mellitus in China. In this study, GQD was fermented with Saccharomyces cerevisiae, and the antidiabetic activities and overall chemical profiles of raw and fermented GQD (FGQD) were systematically compared.
\end{abstract}

Methods: First, the antidiabetic effects of GQD and FGQD on high-fat diet and streptozotocin (STZ)-induced diabetic rats were compared. Then, high-performance liquid chromatography Q Exactive MS was applied for rapid characterization of the chemical components of GQD. Additionally, we proposed an integrated chromatographic technique based untargeted metabolomics identifying differential chemical markers between GQD and FGQD and targeted analysis determining the fermenting-induced quantitative variation tendencies of chemical marker strategy for overall chemical profiling of raw and fermented GQD.

Results: Both GQD and FGQD displayed effects against HFD and STZ-induced diabetes, and FGQD showed a better recovery trend associated with profound changes in the serum lipoprotein profile and body weight gain. In addition, 133 compounds were characterized from GQD. It was demonstrated that the integrated strategy holistically illuminated 30 chemical markers contributed to the separation of GQD and FGQD, and further elucidated the fermenting-induced chemical transformation mechanisms and inherent chemical connections of secondary metabolites. Although there were no new secondary metabolites in FGQD compared with GQD, the amounts of secondary metabolites, which were mostly deglycosylated, were redistributed in FGQD.

\footnotetext{
*Correspondence: qinxm@sxu.edu.cn; sxhpe@163.com

${ }^{\dagger}$ Yan Yan and Chenhui Du contributed equally to this work

${ }^{1}$ Modern Research Center for Traditional Chinese Medicine of Shanxi

University, No. 92, Wucheng Road, Taiyuan 030006, Shanxi, China

${ }^{2}$ School of Traditional Chinese Materia Medica, Shanxi University

of Chinese Medicine, No. 121, Daxue Street, Taiyuan 030619, Shanxi, China

Full list of author information is available at the end of the article
} 
Conclusion: The anti-diabetic activities of GQD could be improved by applying fermentation technology. Moreover, the proposed strategy could serve as a powerful tool for systematically exploring the chemical profiles of raw and fermented formulas.

Keywords: Ge-Gen-Qin-Lian decoction, Fermentation, Untargeted metabolomics, Targeted analysis, Antidiabetic effects

\section{Background}

Herbal fermentation, which began approximately 4000 years ago in China, is used to produce secondary metabolites from plants in bulk by utilizing the metabolic pathways of microorganisms [1]. Fermented medicinal plants and traditional Chinese medicine (TCM) are attracting increasing attention in East Asia, especially in Taiwan and Korea. During the fermentation of TCM, certain glycosides are deglycosylated into small, hydrophobic molecules that may be more efficacious than the original herbal medicines due to increased absorption and bioavailability of the active components in the body [2-5]. Fermented medicinal plants and traditional herbal medicine have been shown to exhibit enhanced antiovarian cancer activity, antioxidative activity, and neuroprotective effects compared to the raw formulas [6-9]. The yeast Saccharomyces cerevisiae (SC) is the most widely used organism for fermentation and has been successfully used for the biotransformation of TCM formula $[4,5,10]$.

Although various beneficial effects of fermentation applied to TCM or medicinal plants have been reported, systematic comparisons of the pharmacological actions and overall chemical profiles of raw and fermented TCM formulas are scarce. TCM is a complex system comprising hundreds of different compounds. Thus, the most critical difficulty is distinguishing and matching herbal biotransformed secondary metabolites in complex microorganism matrixes. Metabolomics, a novel approach for rapidly identifying the global metabolic composition of biological systems, has been widely used for the overall chemical characterization of herbal medicines [11]. Thus, metabolomics analysis could be used to study the effects of fermentation on TCM. In general, the purpose of untargeted metabolomics is to identify statistically significant differences based on unbiased differential analysis of as many signals as possible [12]. By contrast, targeted quantitative metabolomics is intended mainly to accurately determine metabolites in various samples by comparison with authentic compounds to improve the repeatability, comparability and reproducibility of data [13]. Liquid chromatography coupled with mass spectrometry (LC-MS)-based untargeted metabolomic approach can provide global profiles of abundant (up to hundreds of) secondary metabolites by determining their presence, amount and occasionally their structures [14, $15]$ and has been successfully used to study the effects of processing on herbal drugs, such as Rehmanniae Radix and Fructus corni $[15,16]$.

Ge-Gen-Qin-Lian decoction (GQD), a well-known TCM formula, was first recorded in "Treatise on Febrile Diseases" compiled by Zhong-jing Zhang of the Han Dynasty (202 BC-220 AD). GQD consists of four herbs, Pueraria Lobatae Radix, Scutellariae Radix, Coptidis Rhizoma, and Glycyrrhizae Radix et Rhizoma Praeparata cum Melle, in a weight ratio of 8:3:3:2. Extensive chemical studies have shown that flavones (free form and glycosides), flavanones, alkaloids and triterpene saponins are the major compounds in GQD $[17,18]$. Modern pharmacological studies have revealed that GQD has antidiabetic effects in vivo and in vitro [19-22]. GQD is also clinically used to treat type 2 diabetes mellitus (T2DM) [23].

Since GQD and SC have a long history and extensive range of use, their safety and efficacy are demonstrated and widely accepted by the public. Here, GQD was fermented using SC, and the antidiabetic effects of GQD and fermented GQD (FGQD) on high-fat diet (HFD) and streptozotocin (STZ)-induced diabetic rats were compared. An integrated strategy based on untargeted and targeted metabolomic analysis was proposed for the overall chemical profiling of raw and fermented GQD. Finally, the correlations of the biological and chemical differences are discussed.

\section{Methods}

Information on experimental design and resources

The information regarding the experimental design, statistics, and resources used in this study is attached in the minimum standards of reporting checklist (Additional file 1).

\section{Chemicals, materials and reagents}

Acetonitrile (HPLC and MS grade) and methanol (HPLC grade) were purchased from Tedia (Fairfield, USA) and Hanbon (Nanjing, China), respectively. Formic acid (analytical grade) was provided by Aladdin Chemistry Co. Ltd (Shanghai, China). De-ionized water was prepared inhouse by a Milli-Q water purification system (Millipore, MA, USA). Other chemicals and reagents were analytical grade. The chemical reference substances (purity $>98 \%$, 
HPLC-DAD) of $3^{\prime}$-hydroxypuerarin, puerarin, daidzin, daidzein, baicalin, wogonoside, baicalein, wogonin, coptisine, berberine, palmatine, magnoflorine, genistin, genistein, ononin and formononetin were purchased from Chengdu Wei ke-qi Bio-Technology Co., Ltd. (Chengdu, China). Liquiritin, isoliquiritin, liquiritigenin, isoliquiritigenin and glycyrrhizic acid were purchased from Chunqiu Bio-Technology Co., Ltd. (Nanjing, China). Scutellarein (purity $>98 \%$, HPLC-DAD) was isolated, purified and identified in our lab.

Puerariae Lobatae Radix (Gegen), Scutellariae Radix (Huangqin), Coptidis Rhizoma (Huanglian) and Glycyrrhizae Radix et Rhizoma Praepapata Cum Melle (Zhigancao) were purchased from Wan Min pharmacy (Taiyuan, China) and authenticated by Associate Professor Chenhui $\mathrm{Du}$, according to the standard of the Chinese Pharmacopeia (2015 edition). Voucher specimens were deposited in the Modern Research Center for Traditional Chinese Medicine of Shanxi University. SC (CICC 1205) was purchased from the China Center of Industrial Culture Collection (CICC).

\section{Preparation of GQD and FGQD extracts}

Herb pieces of $3200 \mathrm{~g}$ (Gegen:Huangqin:Huanglian:Gan cao $=8: 3: 3: 2)$ were immersed in a 10-fold volume of distilled water $(\mathrm{w} / \mathrm{v})$ for $0.5 \mathrm{~h}$ and then extracted by refluxing two times ( $40 \mathrm{~min}, 30 \mathrm{~min}$ ). For each extract, the decoction was filtered through eight layers of gauze to remove the herbal residue. The two filtrates were combined, condensed under reduced pressure with a rotary evaporator at $70{ }^{\circ} \mathrm{C}$ and evaporated to dryness (yield: $28.6 \%$ ).

Freeze-dried spores of SC were recovered in $25 \mathrm{~mL}$ of potato dextrose (PD) medium and then incubated at $28{ }^{\circ} \mathrm{C}$ on a rotary shaker at $180 \times g$ for $24 \mathrm{~h}$. A $20-\mathrm{mL}$ volume of GQD $\left(0.5 \mathrm{~g} \mathrm{~mL}^{-1}\right.$, crude drug per $\left.\mathrm{g} \mathrm{mL}^{-1}\right)$ was mixed with $30 \mathrm{~mL}$ of distilled water in a $250-\mathrm{mL}$ flask. The substrates of GQD were subjected to autoclaving at $121^{\circ} \mathrm{C}$ for $20 \mathrm{~min}$, then shook evenly and allowed to cool naturally. The sterilized substrates of GQD were inoculated with $2 \%(\mathrm{v} / \mathrm{v})$ recovered SC and incubated at $28{ }^{\circ} \mathrm{C}$ in a shaking incubator $(180 \times g)$. GQD samples were fermented for $48 \mathrm{~h}$ and then evaporated to dryness.

The concentrations of GQD and FGQD were approximately $2 \mathrm{~g} \mathrm{~mL}^{-1}$ (crude drug per $\mathrm{g} \mathrm{mL}^{-1}$ ) for the animal experiments. In addition, the GQD and FGQD extracts for LC and LC-MS analysis were also prepared using the same protocol mentioned above in triplicate.

\section{Animal handing and biochemical parameters related to T2DM measurement}

Male Sprague-Dawley rats (200-220 g) were purchased from Beijing Vital River Laboratories Co., Ltd. (SCXK (Jing) 2014-0013, Beijing, China). The rats were housed at a controlled room temperature of $23 \pm 2{ }^{\circ} \mathrm{C}, 55 \pm 10 \%$ humidity and a 12-h dark-light cycle for 10 days with free access to food and water. Then, 70 rats were randomly divided into two groups: the normal control group $(\mathrm{NC}, \mathrm{n}=10)$ and the diabetic rats group $(\mathrm{n}=60)$. The NC group was fed a regular diet. The diabetic rats group was fed a high-sugar and HFD containing 5\% sucrose, $10 \%$ lard, $5 \%$ yolk powder, $1 \%$ cholesterol, $0.1 \%$ sodium cholate and $78.9 \%$ regular diet. After 4 weeks of dietary intervention, the diabetic rats were fasted for $24 \mathrm{~h}$ and then received STZ $\left(35 \mathrm{mg} \mathrm{kg}^{-1}\right)$ dissolved in citrate buffer $(0.1 \mathrm{M}, \mathrm{pH} 4.5)$ by intraperitoneal injection. The rats in the NC group received an equivalent volume of citrate buffer vehicle. One week after injection, fasting blood glucose (FBG) levels were determined using a drop of blood from the tail vein. Rats with FBG level above $11.1 \mathrm{mM}$ were randomly subdivided into four groups $(\mathrm{n}=13$ for each group): the diabetic model group (DM) and three treatment groups. The treatment groups were fed $0.67 \mathrm{mg} \mathrm{kg}-1$ of metformin hydrochloride (HM), $20 \mathrm{~g} \mathrm{~kg}^{-1}$ of GQD, or $20 \mathrm{~g} \mathrm{~kg}^{-1}$ of FGQD (crude drug per $\mathrm{g} \mathrm{kg}^{-1}$ of body weight) every day for 8 weeks. Body weights were recorded every week, and FBG levels were measured every 2 weeks throughout the experiment.

At the end of the experimental period, the rats were sacrificed under anaesthesia, and blood was immediately collected. Total serum cholesterol (TC), triglycerides (TG), high-density lipoprotein cholesterol (HDL-C) and low-density lipoprotein cholesterol (LDL-C) levels were measured by an ELISA kit (Nanjing jiancheng Bioengineering Institute, Nanjing, China). The fast serum insulin (FINS) concentration was measured using commercial kits (Wa Lan Biotechnology, Shanghai, China). The insulin sensitivity index (ISI) was calculated according to FBG and FINS. The following formula for ISI was used: Ln (1/FBG*FINS) [24]. Homeostasis model assessment-insulin resistance (HOMA-IR) was calculated to measure the insulin sensitivity of the rats fed the experimental diets using the following formula: [FINS $\times \mathrm{FBG}] 22.5^{-1}$ [25]

\section{Statistical analysis}

Data are expressed as the mean \pm S.D. All grouped data were statistically analysed with SPSS 13.0. Statistical significances between means were determined using one-way ANOVA followed by the LSD test of variance homogeneity and Dunnett's T3 test of variance heterogeneity after the normal distribution test. Unless otherwise specified, a value of $p<0.05$ was selected for discriminating significant differences throughout. 


\section{Preparation of standard and sample solutions for HPLC-MS and HPLC analysis}

For HPLC quantification, a mixed stock solution of ten reference substances was prepared at concentrations ranging from 1.0 to $2.5 \mathrm{mg} \mathrm{mL}^{-1}$ in $70 \%$ methanol. A standard working solution of the mixtures was obtained by diluting the stock solutions to the desired concentrations. All solutions were stored at $4{ }^{\circ} \mathrm{C}$ before use.

To obtain sufficient chemical ingredients in the GQD and FGQD extracts, dried extracts $(0.1 \mathrm{~g})$ were accurately weighted and separately extracted in $25 \mathrm{~mL}$ of $70 \%$ methanol $(\mathrm{v} / \mathrm{v})$ for $30 \mathrm{~min}$ by ultrasonication. After adjustment to the initial weight with methanol, $1 \mu \mathrm{L}$ and $10 \mu \mathrm{L}$ of the supernatant solution (obtained by centrifuging at $13,000 \times g$ for $10 \mathrm{~min}$ ) were subjected to LC-MS and LC analysis, respectively. To validate the stability of the sample preparation and instrument, a pooled sample of all samples was prepared as quality control samples (QCs) for LC-MS. QCs were injected six times before the batch process and injected one time every 12 samples during the analysis process.

\section{Untargeted metabolomics analysis by HPLC Q Exactive MS} An HPLC Ultimate ${ }^{\mathrm{TM}} 3000$ instrument coupled with a Q Exactive MS (Thermo Scientific, Bremen, Germany) was used for untargeted metabolomics in this study. Chromatographic separation was performed on an Agilent Poroshell $120 \mathrm{EC}^{-\mathrm{C}_{18}}$ column $(3 \times 100 \mathrm{~mm}$, $2.7 \mu \mathrm{m}$, Agilent, CA, USA). The mobile phase consisted of water containing $0.1 \%(v / v)$ formic acid (A) and acetonitrile (B). The following gradient was used: $0-10 \mathrm{~min}$, $5 \% \mathrm{~B}$ to $17 \% \mathrm{~B} ; 10-12 \mathrm{~min}, 17 \% \mathrm{~B} ; 12-14 \mathrm{~min}, 17 \% \mathrm{~B}$ to $22 \% \mathrm{~B}$; $14-19 \mathrm{~min}, 22 \% \mathrm{~B}$; $19-29 \mathrm{~min}, 22 \% \mathrm{~B}$ to $32 \% \mathrm{~B}$; $29-30 \mathrm{~min}, 32 \% \mathrm{~B}$ to $50 \% \mathrm{~B}$; $30-34 \mathrm{~min}, 50 \%$ B to $90 \%$ B. The column was equilibrated for 5 min prior to each

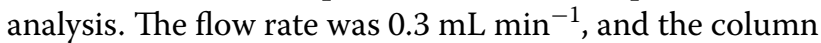
temperature was maintained at $30{ }^{\circ} \mathrm{C}$. The mass spectrometer was operated in both positive and negative ESI full MS-dd-MS/MS acquisition mode with the use of the following parameter settings: spray voltage, $3.5 \mathrm{kV}$; sheath gas: 35 arbitrary units; auxiliary gas: 10 arbitrary units; capillary temperature: $320{ }^{\circ} \mathrm{C}$; S lens RF level: 55; heater temperature: $300{ }^{\circ} \mathrm{C}$. Full scan data were recorded for ions with $m / z 100-1500$ at a resolution of 70,000 (FWHM defined at $m / z 200$ ) in profile format. The automatic gain control (AGC) target values were set at $1 \times \mathrm{e}^{6}$ and $3 \times \mathrm{e}^{6}$ ions, respectively. The injection time was set to $250 \mathrm{~ms}$ in $\mathrm{ESI}^{-}$mode and $100 \mathrm{~ms}$ in $\mathrm{ESI}^{+}$mode. The MS/MS event was triggered when the given precursor ion was detected in an isolation window of $m / z 2.0$. The stepped normalized collision energies (NCE) of the analytes were 10, 30 and 50 .
Targeted quantification analysis by HPLC

Targeted metabolite quantification was performed on a Waters ACQUITY UPLC H-Class system (Milford, MA, USA). Samples were separated on an Agela-MP $\mathrm{C}_{18}$ column $(2.1 \mathrm{~mm} \times 250 \mathrm{~mm}, 5 \mu \mathrm{m}$, Agela, Tianjin, China) maintained at $30{ }^{\circ} \mathrm{C}$. The binary mobile phase consisted of water containing $0.1 \%$ formic acid (A) and acetonitrile (B) at a flow rate of $1.0 \mathrm{~mL} \mathrm{m^{-1 }}$. The optimized gradient elution program was set as follows: $5-20 \% \mathrm{~B}$ (0-25 min), 20\% B (25-30 min), 20-22\% B (30-35 min), $22-40 \%$ B (35-55 min), 40-63\% B (55-65 min), 63-80\% B (65-70 min). The UV signals from two separate channels of $254 \mathrm{~nm}$ and $276 \mathrm{~nm}$ were recorded.

\section{Data processing and analysis}

Data from the HPLC Q Exactive MS acquisition and processing were used for chemical profile analysis using Xcalibur $^{\mathrm{TM}} 2.2$ (Thermo Fisher). The untargeted metabolomics analysis was conducted by using Compound Discovery (version 1.2.1, Thermo SCIEX), and the detailed workflow is shown in Additional file 2: Figure S1. The multivariate data matrix was introduced into SIMCA-P (Version 13.0, Umetrics AB, Umea, Sweden) software for "unsupervised" principal component analysis (PCA) and "supervised" orthogonal projection to latent structurediscriminant analysis (OPLS-DA). All variables were UVscaled for PCA and Pareto-scaled for OPLS-DA.

\section{Results}

\section{Antidiabetic effect}

As shown in Fig. 1, the body weight of the diabetic rats decreased significantly compared with the NC group after STZ injection $(p<0.01)$. HM reversed the diabetes-induced body weight decrease from the 6th week $(p<0.05)$, whereas FGQD significantly reversed the body weight decrease from the 7 th and 8th weeks $(p<0.01$, $p<0.05)$. However, no significant $(p>0.05)$ effect was observed for the GQD group, suggesting that GQD had

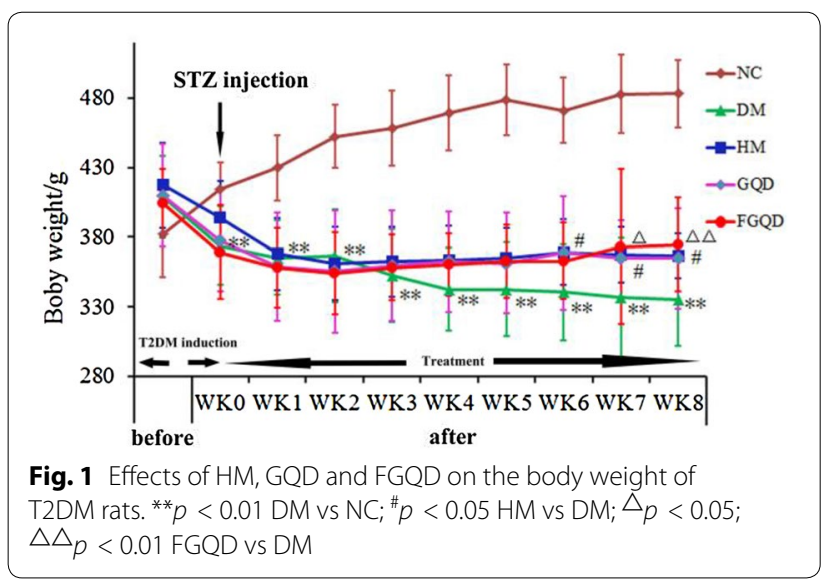




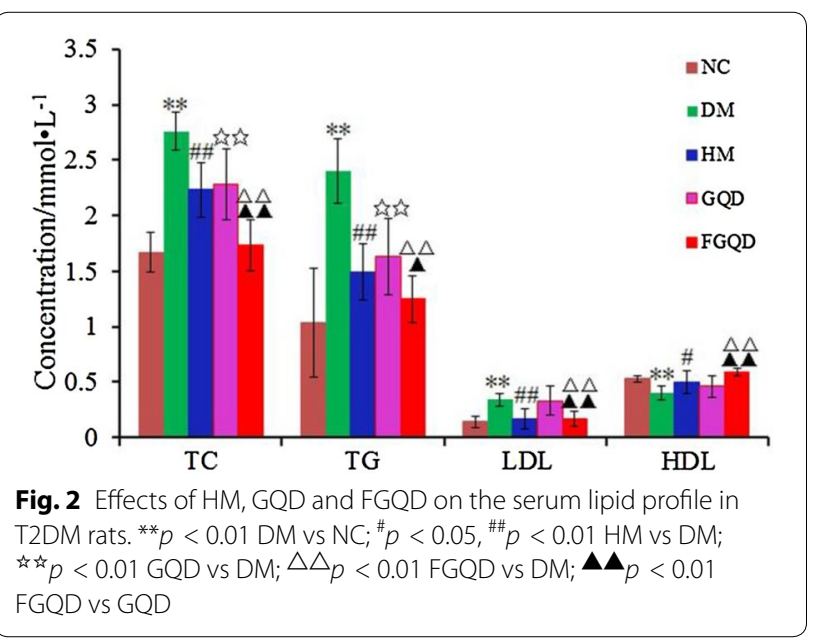

Table 1 Effects of HM, GQD and FGQD on FINS, ISI and HOMA-IR of T2DM rats

\begin{tabular}{|c|c|c|c|}
\hline Group & FINS (mIU/L) & ISI & HOMA-IR \\
\hline $\mathrm{NC}$ & $4.92 \pm 0.74$ & $-3.38 \pm 0.24$ & $1.33 \pm 0.30$ \\
\hline DM & $9.88 \pm 0.58^{* *}$ & $-5.24 \pm 0.22^{* *}$ & $8.59 \pm 1.75^{* *}$ \\
\hline HM & $7.17 \pm 0.54^{\# \#}$ & $-4.47 \pm 0.36^{\# \#}$ & $3.98 \pm 1.07^{\# \#}$ \\
\hline GQD & $6.78 \pm 0.35$ 不的 & $-4.52 \pm 0.23$ 不的 & $4.18 \pm 0.95$ 年的 \\
\hline FGQD & $5.86 \pm 0.55 \triangle \triangle \boldsymbol{\Delta} \mathbf{\Delta}$ & $-4.26 \pm 0.18 \triangle \triangle$ & $3.20 \pm 0.60^{\triangle}$ \\
\hline
\end{tabular}

NC normal control, DM diabetic model, HM metformin hydrochloride, ISI insulin sensitivity index, FINS fast serum insulin, HOMA-IR homeostasis model assessment-insulin resistance, T2DM type 2 diabetes mellitus

${ }^{* *} p<0.01 \mathrm{DM}$ vs NC; ${ }^{\prime} p<0.05, \# \#<0.01 \mathrm{HM}$ vs DM; ; $p<0.01 \mathrm{GQD}$ vs DM; $\triangle \triangle_{p}<0.01$ FGQD vs DM; $\boldsymbol{\Delta} \mathbf{\Delta}_{p}<0.01$ FGQD vs GQD

no significant effect on weight gain. As shown in Additional file 2: Figure S2, the FBG level was significantly increased in the diabetic rats compared to the NC group $(p<0.01)$ and was decreased in all drug-treated groups from the 4th week $(p<0.01, p<0.05)$ after the injection of STZ. Although no significant difference was observed among the drug-treated groups $(p>0.05)$, the diabetic rats in FGQD showed a better trend of recovery. Rats in the model group had significantly higher levels of TC and TG $(p<0.01)$ than those in the NC group, and these levels were reduced in all drug-treatment groups $(p<0.01)$ (Fig. 2). Notably, the levels of TC and TG were significantly lower in the FGQD group than in the GQD group $(p<0.01)$ (Fig. 2). In addition, the treatments with HM and FGQD reversed the up-regulation of LDL and down-regulation of HDL in the diabetics rats group to the control level, whereas no significant $(p>0.05)$ effect was observed for GQD (Fig. 2). As shown in Table 1, the diabetic rats showed significant increases in FINS and HOMA-IR $(p<0.01)$ and a decrease in ISI $(p<0.01)$ compared with the NC group. After 8 weeks of drug administration, the levels of FINS, ISI and HOMA-IR were reversed compared with the DM group $(p<0.01)$. In addition, a notable difference in FINS level was observed in the FGQD group $(p<0.01)$ compared with the GQD group. In short, the body weight gain and the regulation of the levels of FINS, TC, TG, LDL and HDL in the FGQD group were significantly better than those in the GQD group $(p<0.01)$, but there were no significant differences in FBG, ISI and HOMA-IR levels between GQD and FGQD. These results suggested that FGQD had better therapeutic effect against diabetes than GQD.

\section{Characterization of the chemical constituents in the GQD extract}

Since herbal medicines are generally taken as a decoction, we focused on boiled water extracts of GQD and their fermentation. The structural characterization of compounds in GQD is an essential step in identifying and matching those compounds with their secondary metabolites obtained through biotransformation. All known compounds were identified by comparison with chemical standards. For unknown compounds, structures were tentatively characterized based on retention time and MS spectra by referring to the previous literature. Finally, assignments of all compounds were further conducted by comparing the corresponding extracted ion chromatography (EIC) of GQD with those of the individual herbs. In total, 133 compounds were rapidly identified or tentatively characterized; these compounds were divided into six structural types. The detailed information, including retention times, accurate $m / z$, ppm errors, characteristic fragment ions, identified names and formulas, are summarized in Table 2, Additional file 2: Figure S3. Notably, two compounds were identified for the first time in GQD: 6-D-xylose-genistin and kuzubutenolide A.

\section{Isoflavone glycosides}

In total, 17 isoflavone $C$-glycosides and 15 isoflavone $O$-glycosides were identified as the dominant compounds from Gengen in GQD (Additional file 2: Figure S4A). P6, P11, P18, P26 and P34 were unambiguously identified by comparison with reference compounds. According to the MS/MS analysis of these authentic compounds, isoflavone $O$-glycosides (P18, P26 and P34) showed dominant aglycone ions at $m / z 255,271$ and 269 , respectively, due to the loss of a glucose group (162 Da). By contrast, isoflavone $C$-glycosides (P6 and P11) were hardly cleaved under the same conditions and shared the common principal fission pattern of successive or simultaneous losses of $\mathrm{CO}, \mathrm{CHO}$ and $\mathrm{CH}_{2} \mathrm{O}$ groups caused by cleavage of the $\mathrm{C}$-ring. Consequently, the major fragmentation behaviours were summarized and then applied as rules to 


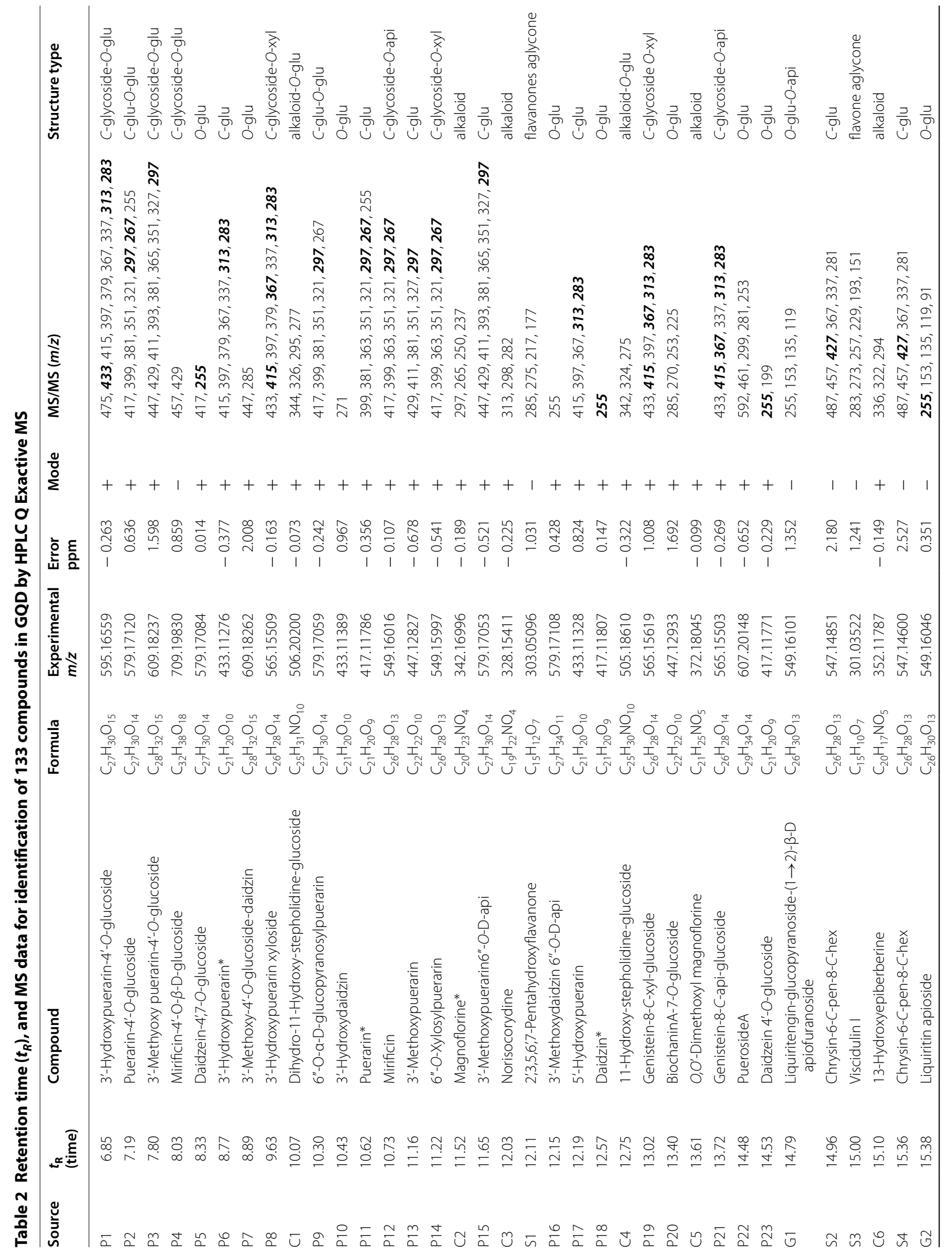




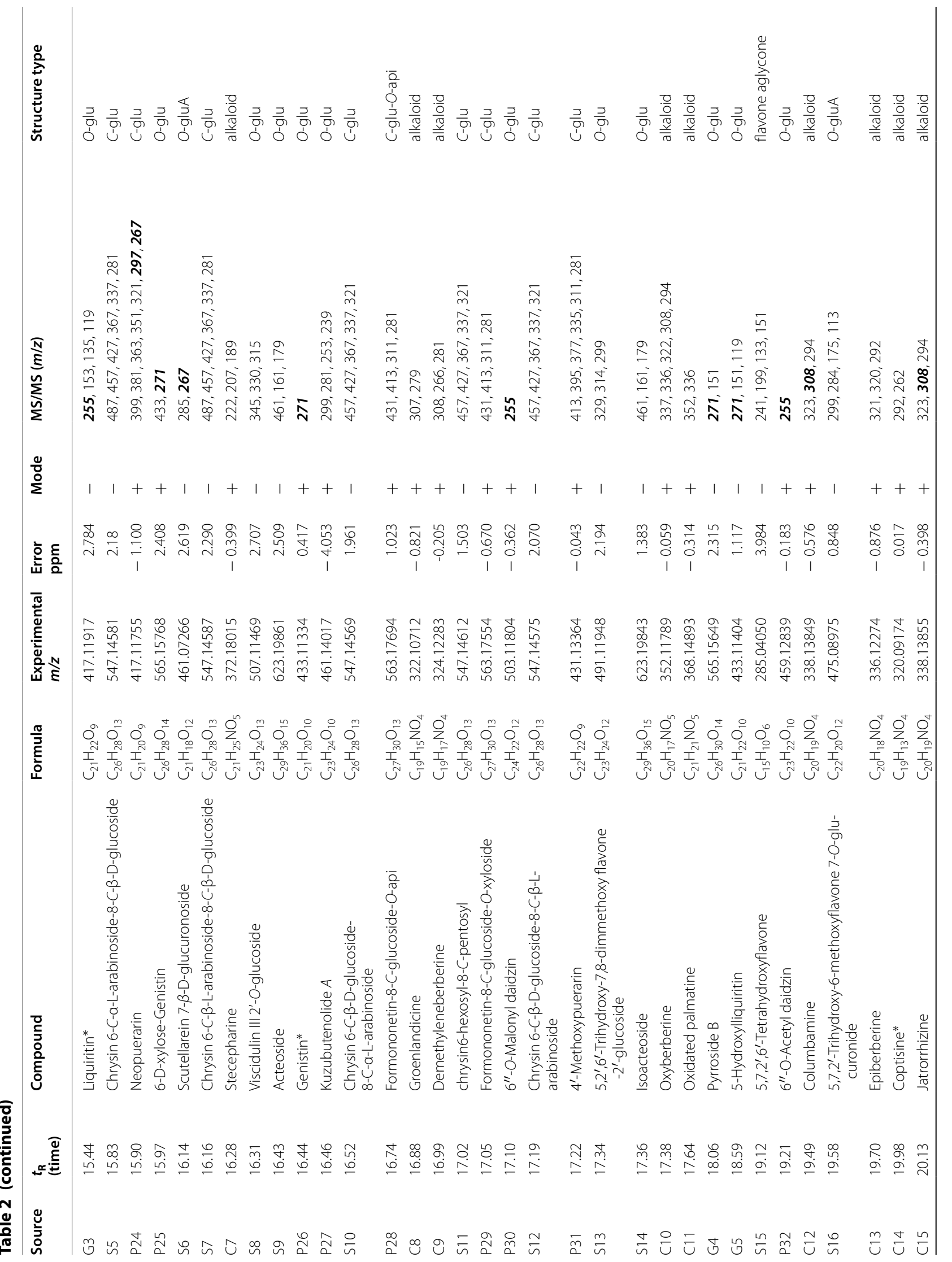




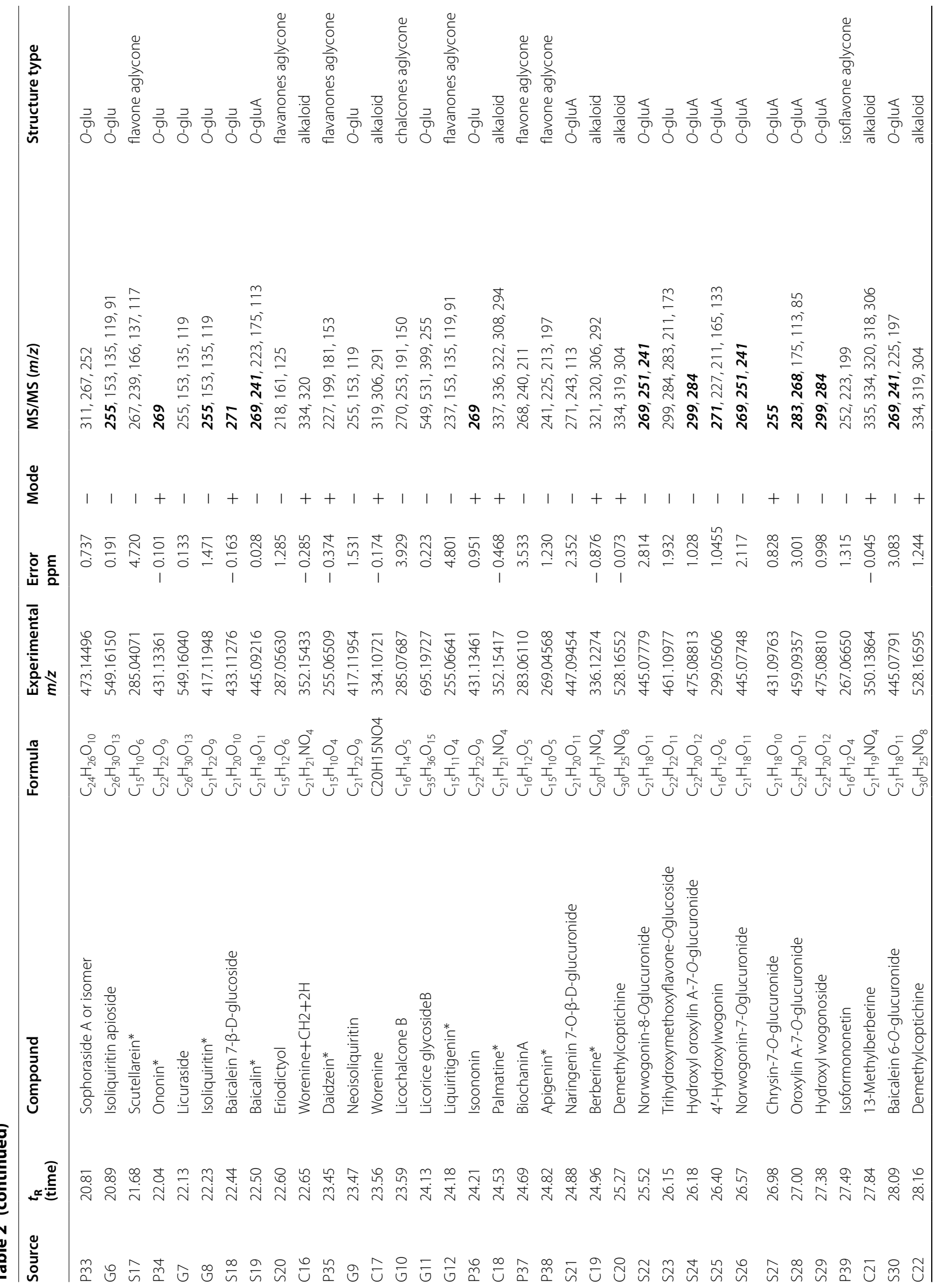




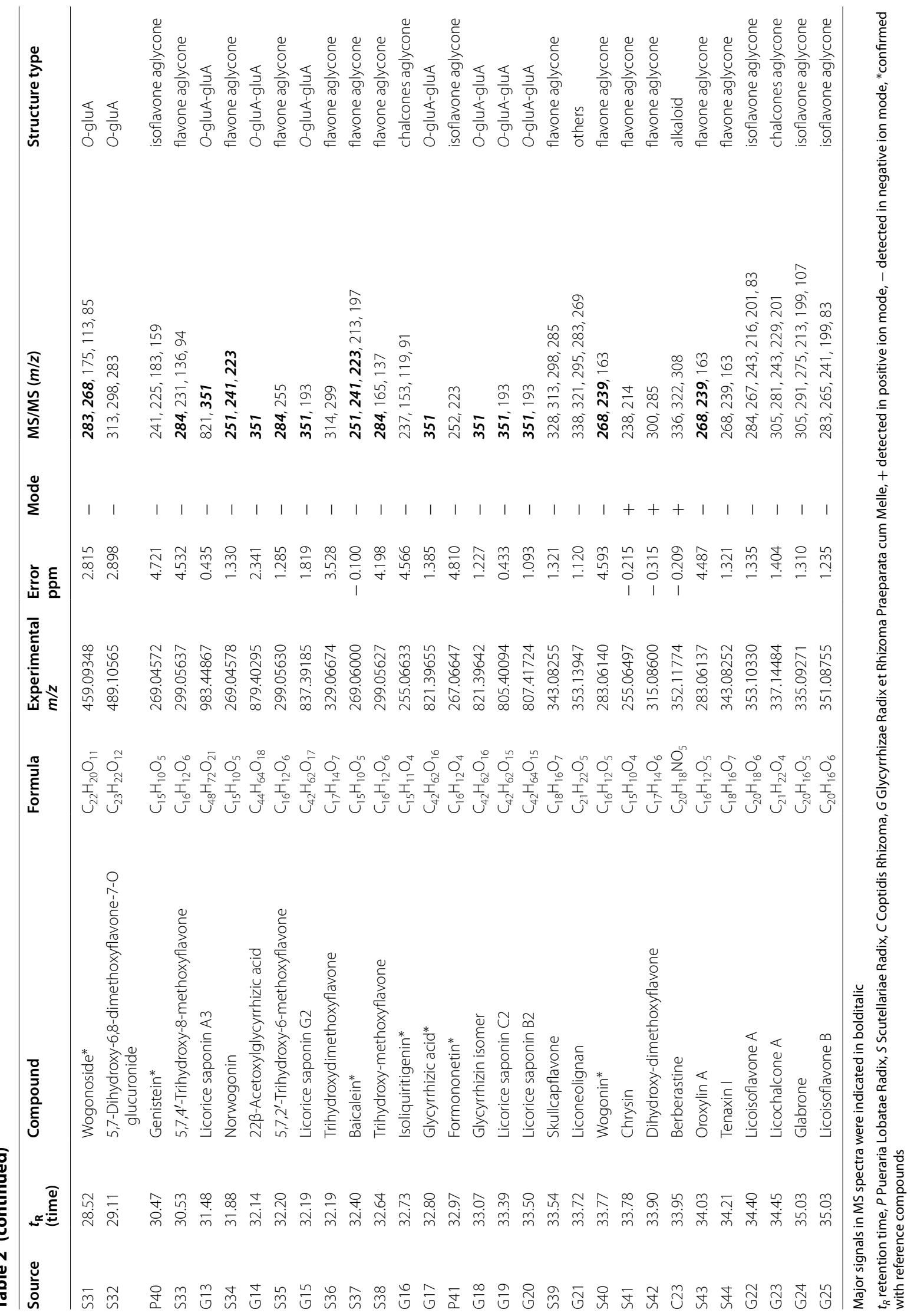


elucidate the structures of the other 27 unknown compounds with the same basic skeleton [18, 26, 27]. Among them, P25 showed a precursor ion with $\mathrm{m} / z 565.15509$ and further fragmented into the characteristic ion at $m / z 271$, corresponding to $[\mathrm{M}+\mathrm{H}-\mathrm{xyl} / \mathrm{api}-\mathrm{glu}]^{+}$. More importantly, P25 was tentatively deduced as 6-D-xylosegenistin in GQD for the first time.

\section{Flavone glycosides}

The occurrence of flavone $O$-glucuronides is less common in plants. Previously published studies have thoroughly summarized the fragmentation pathways of flavonoids $O$-glucuronides in Huangqin [28]. As characteristic components, a total of 12 flavone $O$-glucuronides (S6, S16, S19, S22, S24, S26, S27, S28, S29, S30, S31 and S32) all from Huangqin were identified and tentatively characterized in GQD (Additional file 2: Figure S4B) [28-33]. Moreover, S2, S4, S5, S7, S10, S11 and S12 were tentatively characterized as flavone $C$-glycosides. In addition, S8, S13, S18 and S23 were excluded from flavone $O$-glucuronides by analysing the MS/MS spectra and then were finally identified as flavone $O$-glycosides [33].

In addition, six flavanones glycosides and five chalcones glycosides were putatively characterized in GQD (Additional file 2: Figure S4C). Among them, G3 and G8 were identified as liquiritin and isoliquiritin, respectively, by comparison with reference standards, and the others from Gancao were characterized by analysing their MS/ MS spectra [32, 34]. In addition, S21 was characterized as a flavanone glycoside from Huangqin.

\section{Free flavones}

In total, 30 free flavones were tentatively assigned and could be further divided into isoflavones (8), flavones (16), flavanones (3) and chalcones (3) in GQD (Additional file 2: Figure S4D). P35, P40 and P41 were confirmed by comparison with reference standards. P37 and P39 from Gegen and G22, G24 and G25 from Gancao were tentatively characterized as isoflavone aglycones by analysing the MS/MS spectra [2,32]. In addition, the flavones comprised 16 compounds from Huangqin. Baicalein (S37) produced characteristic ions with $\mathrm{m} / z 251,241$ and 223 by loss of $\mathrm{H}_{2} \mathrm{O}$ and CO. Wogonin (S40), a methoxylated flavonoid, presented a deprotonated ion $[\mathrm{M}-\mathrm{H}]^{-}$at $\mathrm{m} / \mathrm{z}$ 283.06140 and characteristic fragment ions with $\mathrm{m} / z 268$ and 239. In addition, a low signal intensity ion with $\mathrm{m} / \mathrm{z}$ $163\left({ }^{0,2} \mathrm{~A}^{-}\right)$through Retro-Diels-Alder (RDA) cleavage was observed. Thus, the other 14 flavones in the complex mixtures were characterized based on the literature $[28,33]$. In negative ion mode, liquiritigenin (G12) and isoliquiritigenin (G16), a pair of isomers, showed fragmentation patterns associated with RDA cleavage at $\mathrm{m} / \mathrm{z}$ 135 or 119 . Thus, S1, S20, G10 and G23 were tentatively characterized according to the above mentioned MS behaviours [28].

\section{Alkaloids}

A total of 23 alkaloids from Huanglian were characterized based on positive ion mode mass spectra (Additional file 2: Figure S4E). Three benzylisoquinoline alkaloids, i.e., coptisine, palmatine and berberine, were identified by comparison with their authentic standards and the production of one or multiple common small fragments such as $\mathrm{H}_{2} \mathrm{O}, \mathrm{CH}_{3}$ and $\mathrm{C}_{2} \mathrm{H}_{6} \mathrm{~N}$, respectively. Based on these rules, $\mathrm{C} 6, \mathrm{C} 8, \mathrm{C} 9, \mathrm{C} 10, \mathrm{C} 11, \mathrm{C} 12, \mathrm{C} 13, \mathrm{C} 15, \mathrm{C} 16$, $\mathrm{C} 21$ and $\mathrm{C} 23$ were observed and further tentatively characterized by analysing characteristic ions [35, 36]. Magnoflorine, an aporphinoid alkaloid, exhibited a precursor ion at $m / z 342.16996$ and characteristic ions at $m / z 297$, 265, 250 and 237. Similarly, C4 and C5 were tentatively identified as aporphinoid alkaloids. The others $(\mathrm{C} 1, \mathrm{C} 3$, $\mathrm{C} 7, \mathrm{C} 17, \mathrm{C} 20$ and C22) were characterized by comparison to the literature [27].

\section{Triterpene saponins}

Triterpene saponins were the other characteristic constituents from Gancao. In total, six triterpene saponins were putatively identified (Additional file 2: Figure S4C). Glycyrrhizic acid (G17 or G18) presented an $[\mathrm{M}-\mathrm{H}]^{-}$ ion with $m / z 821.39655$ and characteristic fragment ions at $m / z 351$ and 193 [32]. G13, G14, G15, G19 and G20 showed characteristic ions similar to those of glycyrrhizic acid and were tentatively characterized according to the literature [18].

\section{Others}

In addition to the major compounds described above, atypical structures were also found in GQD (Additional file 2: Figure S4C). P22 and P33, which belong to aromatic glycosides, were identified as pueroside $\mathrm{A}$ and sophoroside A or their isomers [26]. P27 showed an $[\mathrm{M}+\mathrm{H}]^{+}$ion at $m / z 461.14017$ with $\mathrm{MS}^{2}$ characteristic peaks at $m / z 299,281,253$ and 239 and was tentatively identified as kuzubutenolide A in GQD for the first time [37]. In addition, S9 and S14 were tentatively identified as isomers of acteoside and isoacteoside [12, 38], and P38 and G21 were also tentatively characterized by comparison with the literature [33].

\section{Multivariate statistical analysis}

To identify chemical markers distinguishing GQD and FGQD samples, the negative and positive ion mode data detected by HPLC Q Exactive MS were simultaneously used for global analysis. Visual inspection of the chromatograms for GQD and FGQD indicated that the fermentation process induced obviously different peak 

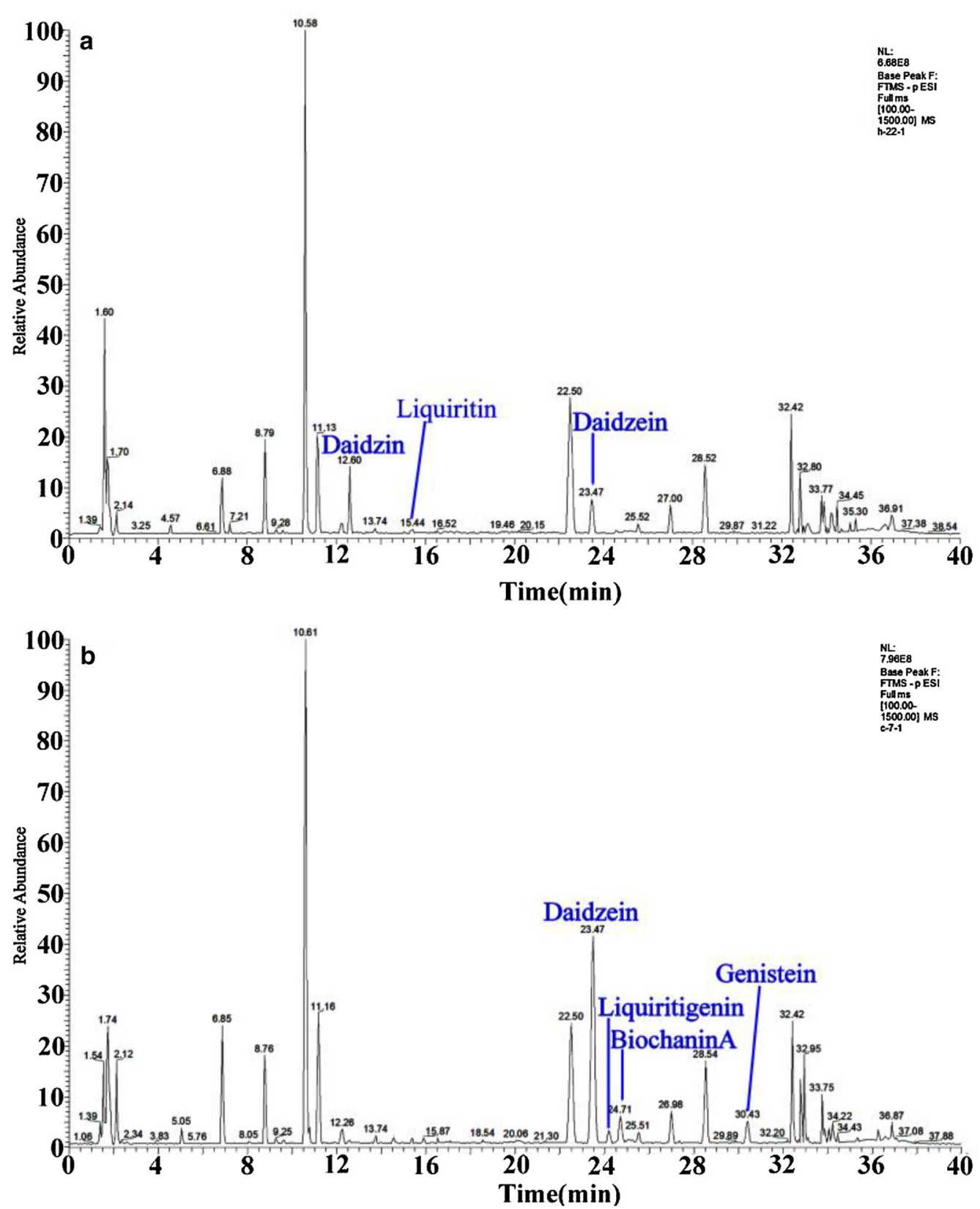

Fig. 3 Typical basic peak ion chromatograms obtained by HPLC Q Exactive MS. a GQD; b FGQD. All chromatograms were obtained in negative ion mode

intensities; that is, FGQD contained more daidzein, liquiritigenin, genistein, and biochanin A and less daidzin and liquiritin than GQD (Fig. 3). Multivariate statistical analysis was subsequently applied to further reveal the minor differences between GQD and FGQD. In the PCA score plot (Additional file 2: Figure S5A, B) generated by $\mathrm{PC} 1(46.2 \%)$ and $\mathrm{PC} 2(17.9 \%)$ for positive ion mode and PC1 (51.1\%) and PC2 (17.9\%) in negative ion mode, clear separation can be observed between GQD and FGQD. Then, OPLS-DA was further performed to process the secondary metabolome data between the GQD and FGQD groups by S-plot and VIP-value analysis. The model fit parameters were 0.999 for $\mathrm{R}^{2} \mathrm{Y}$ (cum) and 0.971 for $Q^{2}$ (cum) for positive ion mode and 0.999 for $\mathrm{R}^{2} \mathrm{Y}$ (cum) and 0.987 for $\mathrm{Q}^{2}$ (cum) for negative ion mode, respectively, suggesting that the OPLS-DA model exhibited good fitness and predictability. In the S-plots, each point represented an ion $t_{R^{-}} m / z$ pair, whereas the 

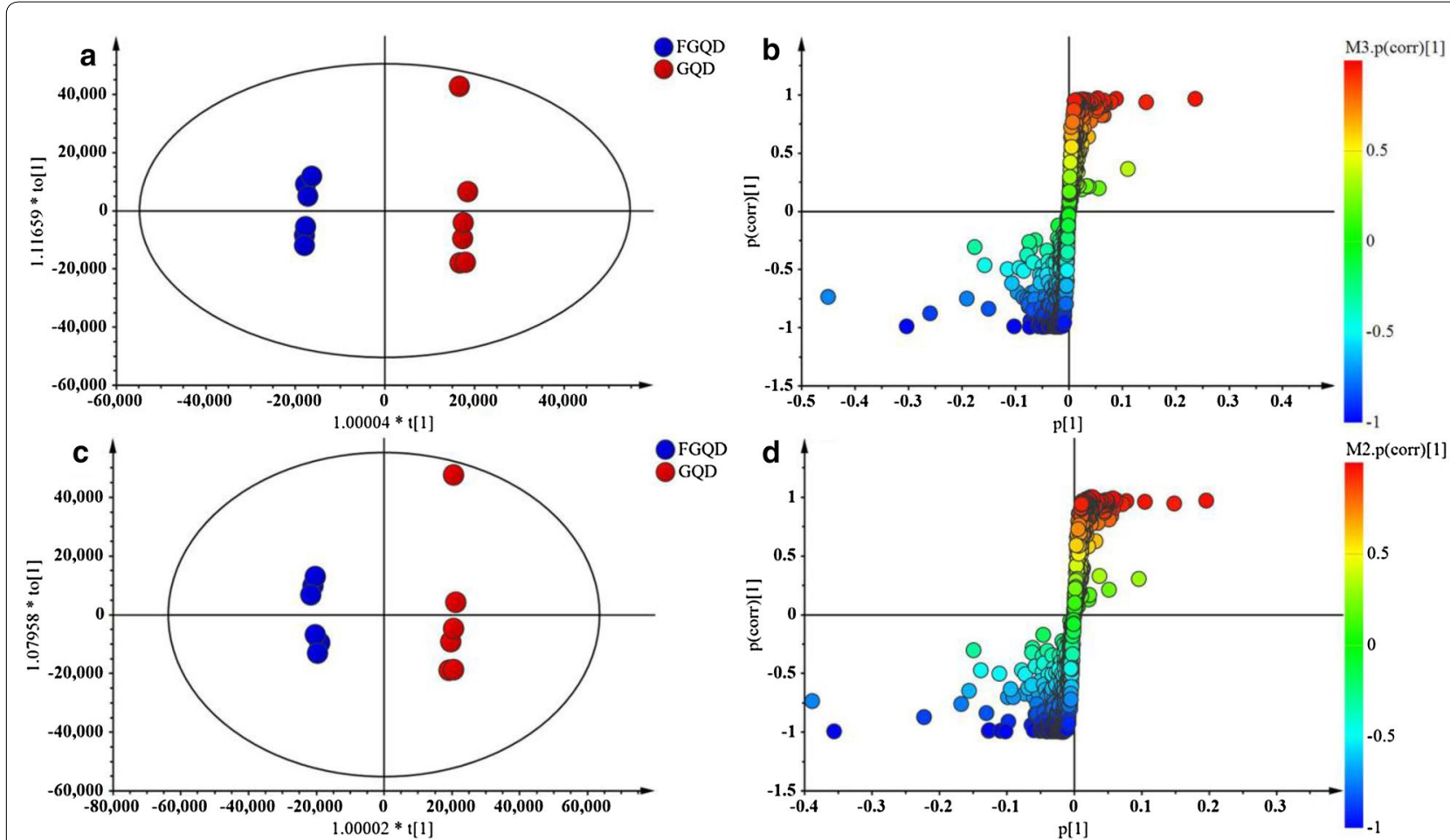

Fig. 4 OPLS-DA score plots $(\mathbf{a}, \mathbf{c})$ and S-plots (b, d) between GQD and FGQD. $\mathbf{a}$ and $\mathbf{c}$ present data in positive ion mode; $\mathbf{b}$ and $\mathbf{d}$ present data in negative ion mode

distances of the pair points from the mean centre indicate the contribution of the variables in discriminating the GQD and FGQD groups (Fig. 4a, b). The VIP-value threshold cut-off of the variables was set to one, and thus 83 and 117 variables were finally screened in LC/MS $\left(\mathrm{ESI}^{+}\right)$and LC/MS $\left(\mathrm{ESI}^{-}\right)$, respectively. Among them, 25 variables were identified in both ion modes. Three variables and two variables were identified in negative ion mode and positive ion mode, respectively. Thus, 30 compounds that had different intensities between GQD and FGQD were detected.

To maximize the understanding of the effect of fermentation on GQD, the mean peak areas and the t-test results for the significant differences in the $30 \mathrm{com}-$ pounds from GQD and FGQD are shown in Figs. 5, 6. As shown in Fig. 5a1, the mean peak areas of free flavones (P35, P37, P40 and G12) were larger in FGQD than in GQD $(p<0.001)$, whereas the mean peak areas of their corresponding $O$-glycosides (P5, P18, P20, P26, G2 and G3) were smaller in FGQD than in GQD $(p<0.001$, $p<0.05)$, indicating that $O$-glycoside hydrolysis occurred during fermentation processing (Fig. 5a2). P23 could also be transformed to P35 by $O$-glycoside hydrolysis. In addition, P10 and P34 contained abundant hydroxyl and methyl and were deduced to possibly produce P18 by dehydroxylation or demethylation. Actually, a marked decline in the level of P34 was also observed $(p<0.01)$ (Fig. 5a1), however, its corresponding aglycone P41 was not obviously altered in FGQD, which might be due to a dynamic equilibrium between their formation (from $\mathrm{O}$-glycoside hydrolysis) and further transformation (e.g., demethylation). By contrast, $C$-glucosides appeared to be more difficult to transform by $\mathrm{SC}$, since five $C$-glucosides (P6, P11, P13, P14 and P24) were detected in FGQD (Fig. 5b1). Their significant increasing trend was probably caused by the hydrolysis of low contents of puerarin $\mathrm{C}$-glucoside- $\mathrm{O}$-glucoside derivatives, such as $\mathrm{P} 1, \mathrm{P} 2$, P3, P4, P8, P12 and P15 (Fig. 5b2). O-C glycoside bonds have been reported to be the main effective target of $\beta$-glucosidase [13], in agreement with our results that puerarin (P11) and its derivatives were difficult to hydrolyse by $\beta$-glucosidase.

As shown in Fig. 6a1, the remarkable increase in the level of flavone aglycone (S43) was potentially due to hydrolysis of the corresponding flavone $O$-glucuronide (S28), which contains a $6-\mathrm{OCH}_{3}$ group $(p<0.001)$. S31, which contains an $8-\mathrm{OCH}_{3}$ group, was more difficult to transform by hydrolysis by SC but was easier to produce from S25 by dehydroxylation (Fig. 6a2). Although a different strain of yeast was used, the current findings are still in agreement with those in a previous study [39]. Notably, the increasing trend of S37 is likely partially 
Pan et al. Chin Med <wide> (2018) 13:54

Page 13 of 18

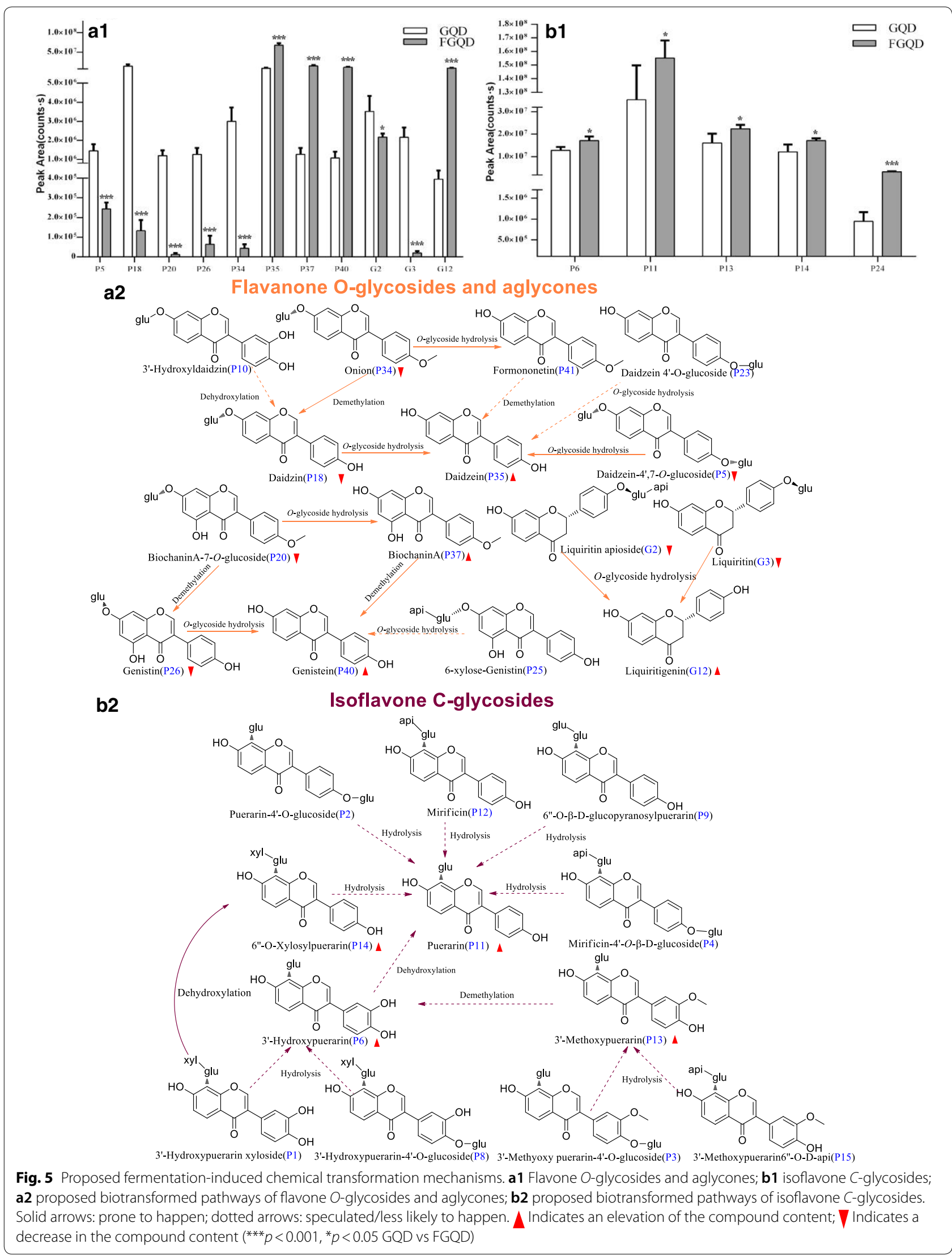



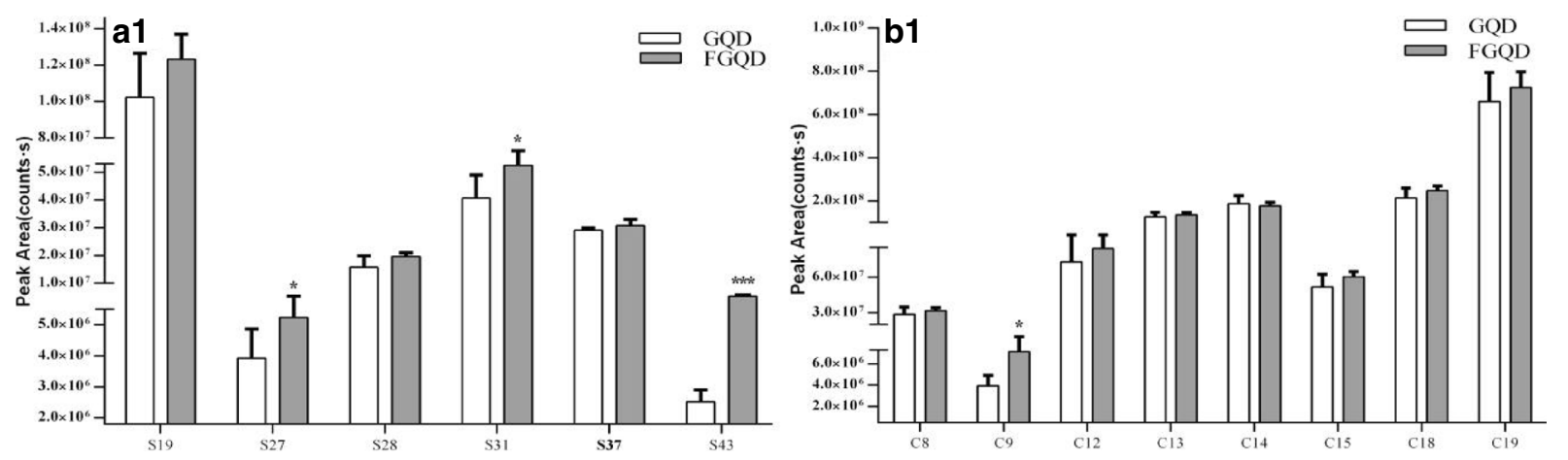

a2

Flavonoid O-glucuronides

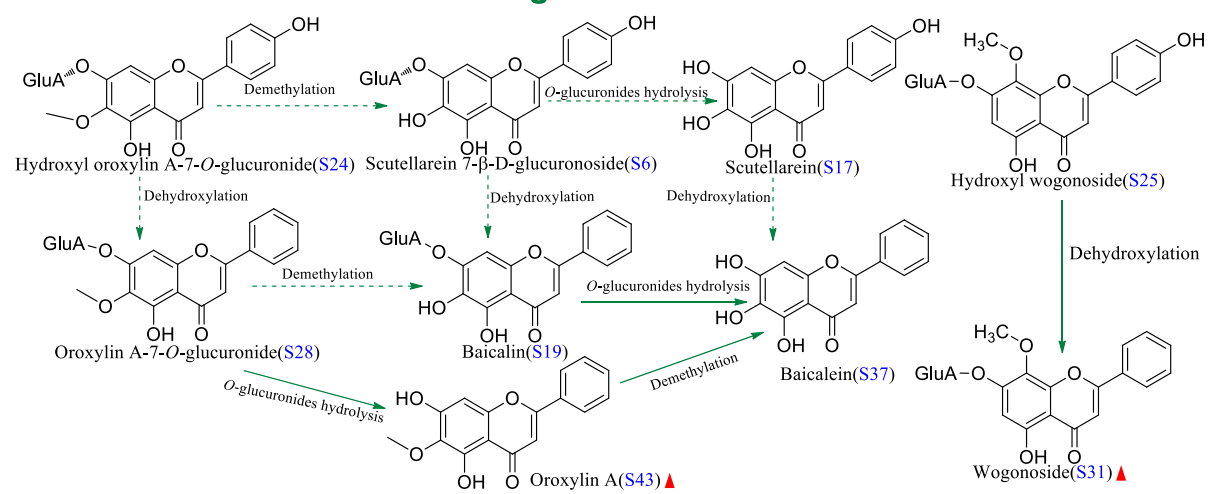

b2

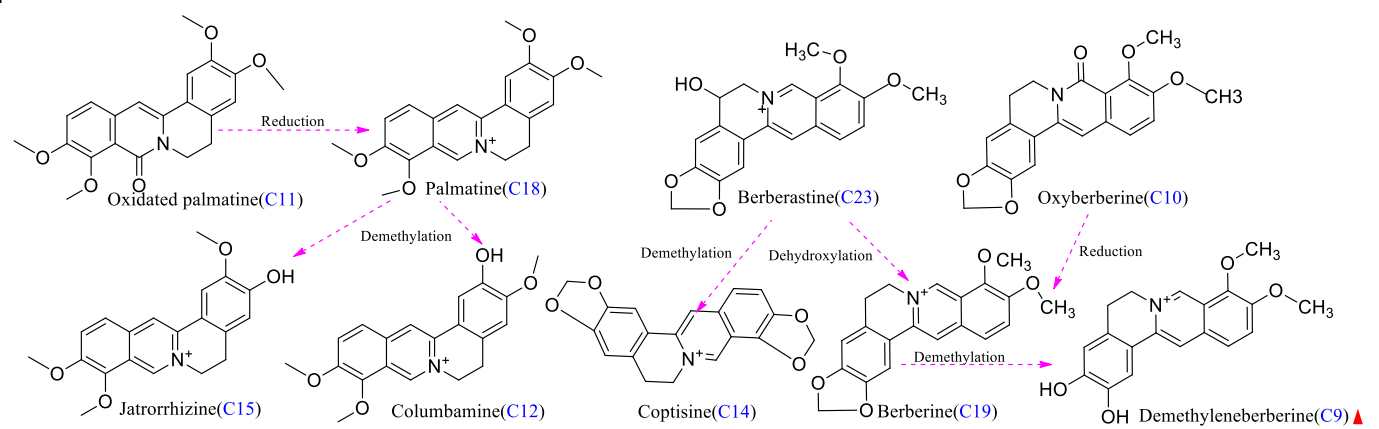

Fig. 6 Proposed fermentation-induced chemical transformation mechanisms. a1 Flavone O-glucuronides; b1 alkaloids; a2 proposed biotransformed pathways of flavone O-glucuronides; b2 proposed biotransformed pathways of alkaloids. Solid arrows: prone to happen; dotted arrows: speculated/less likely to happen. Indicates an elevation of the compound content; $\boldsymbol{\nabla}$ Indicates a decrease in the compound content $\left({ }^{* * *} p<0.001,{ }^{*} p<0.05\right.$ GQD vs FGQD)

responsible for the hydrolysis reactions of the corresponding compound (S19) (Fig. 6a2). A previous study demonstrated that Escherichia (E.) coli $\beta$-glucuronidases could hydrolyse glucuronic acid at the 7-position if the structure contains a 6-OH group [39]. Other metabolic reactions for flavone-O-glucuronides, including demethylation and dehydroxylation, were also deduced.

Due to the lack of a free hydroxyl group, alkaloids are demethylated to form free hydroxyl groups by SC [36]. In this study, a significant increase in demethyleneberberine (C9) was observed in FGQD compared to GQD $(p<0.05)$, which probably contributed to the demethylation of C19 during fermentation processing (Fig. 6b1, b2). There were no significant differences in the other benzylisoquinoline alkaloids between GQD and FGQD $(p>0.05)$, thus indicating that the contents of these molecules remained stable during the fermentation process.

\section{Targeted quantification analysis}

As mentioned above, the untargeted metabolomic studies indicated that isoflavone $O$-glycosides, flavone $O$-glycosides, flavone $O$-glucuronides and alkaloids were 
Table 3 Contents of 10 chemical markers in GQD and FGQD by SC $\left(\mathrm{mg} \mathrm{g}^{-1}, \mathrm{n}=3\right)$

\begin{tabular}{lcl}
\hline Compound & \multicolumn{1}{l}{$\mathbf{h}$} & $\mathbf{4 8} \mathbf{h}$ \\
\hline Puerarin & $20.30 \pm 0.05$ & $23.57 \pm 0.02^{*}$ \\
Daidzin & $3.67 \pm 0.08$ & n.d.*** \\
Daidzein & $0.50 \pm 0.02$ & $3.80 \pm 0.01^{* * *}$ \\
Liquiritin & $0.80 \pm 0.06$ & $0.48 \pm 0.02^{*}$ \\
Liquiritigenin & $0.17 \pm 0.05$ & $0.50 \pm 0.01^{*}$ \\
Coptisine & $2.23 \pm 0.12$ & $2.68 \pm 0.003$ \\
Palmatine & $2.01 \pm 0.03$ & $2.36 \pm 0.15$ \\
Berberine & $7.70 \pm 0.03$ & $8.10 \pm 0.02$ \\
Baicalin & $10.80 \pm 0.02$ & $11.85 \pm 0.01$ \\
Baicalein & $1.26 \pm 0.04$ & $1.27 \pm 0.03$ \\
\hline
\end{tabular}

The content is expressed in units of $\mathrm{mg} / \mathrm{g}$. n.d. indicates under the LOQ. ${ }^{*} p<0.05$, *** $p<0.001 \mathrm{FGQD}$ vs GQD

potential chemical markers for distinguishing GQD and FGQD. Thus, three $O$-glycosides (daidzin, baicalin and liquiritin), one $C$-glycoside (puerarin), three flavones (daidzein, liquiritigenin, and baicalein), and three alkaloids (coptisine, berberine and palmatine) were quantitatively determined as examples to illustrate the effects of processing (Additional file 2: Figure S3, Table S1). Their content changes in GQD and FGQD are summarized in Table 3. As expected, fermentation processing significantly depleted liquiritin ( $O$-glycoside) from $0.80 \pm 0.06 \mathrm{mg} \mathrm{g}^{-1}$ to $0.48 \pm 0.02 \mathrm{mg} \mathrm{g}^{-1}(p<0.05)$, whereas daidzin was not even detectable in FGQD $(p<0.001)$ after fermentation with SC. Interestingly, the concentrations of daidzein and liquiritigenin (free flavones) in FGQD were greatly enhanced $(p<0.001$, $p<0.05$, respectively). In addition, an obvious increase in the level of puerarin (isoflavone $C$-glycoside) was observed until the end of fermentation. Regarding alkaloids, the contents of coptisine, palmatine and berberine remained relatively stable $(p>0.05)$. Moreover, there was a slight increasing trend for baicalin (flavone $O$-glucuronide), whereas no significant difference was found between GQD and FGQD. Interestingly, the quantitative results revealed an increasing trend for baicalein $(p>0.05)$ did not correspond to the results of the untargeted studies, which showed a significant increase in the content of baicalein in FGQD compared with GQD $(p<0.05)$.

\section{Discussion}

GQD is a well-known TCM formula that has been reported to display anti-diabetic properties in the clinic [20]. In the present study, we investigated the efficiency of FGQD and confirmed that fermentation actually enhanced the anti-diabetic activities of GQD in vivo in diabetic rats induced by HFD and STZ. The present results suggested that GQD had no significant effect on weight gain, in agreement with a previous study [19], whereas FGQD showed a significant reversed trend. In addition, our study indicated that the level of FBG was conspicuously decreased, accompanied by decreases in serum TG, TC, LDL-C and FINS and increased HDL-C after GQD treatment, consistent with previous work [21]. FGQD exerted greater regulatory effects on the levels of TC, TG, LDL-C, HDL-C and FINS compared to GQD. Thus, both GQD and FGQD displayed effects against HFD and STZ-induced diabetes, and FGQD showed a better recovery trend associated with profound changes in the serum lipoprotein profile and body weight gain. These findings further suggest that fermentation can play a key role in the search for therapeutically useful drugs. Given the pharmacologically decisive roles of the involved ingredients, chemical transformations might significantly contribute to the therapeutic differences between GQD and FGQD. Thus, the chemical profiles of GQD and FGQD were further systematically compared using the proposed integrated strategy based on untargeted and targeted metabolomic analysis.

In this study, 133 secondary metabolites analysed using UPLC-Q Exactive MS were identified and characterized by comparison with standard references and the literature. Then, untargeted metabolomics was performed to find statistically significant differences between GQD and FGQD groups via OPLS-DA S-plot and VIP-value analysis. The OPLS method is a modification of the PLS method with a multivariate pre-processing filter called orthogonal signal correction (OSC). The OSC filter removes uncorrelated signals to provide information on the within-class variation [40]. Overall, 30 potential chemical markers contributed to the separation of GQD and FGQD, and the mechanisms of the processing-induced chemical transformation of the secondary metabolites were further elucidated. Although there were no new secondary metabolites in FGQD compared with GQD, the amounts of these secondary metabolites were redistributed in FGQD. Deglycosylation reaction by stepwise cleavage of the sugar moieties was considered the main metabolic pathway. Other chemical reactions, i.e., dehydration, demethylation and reduction, were also potentially implicated in the processing. These chemical transformations should mainly contribute to the fluctuation in the contents of isoflavone $\mathrm{O}$-glycosides and flavone $\mathrm{O}$-glucuronides due to processing. These results for the in vitro biotransformation of GQD by SC demonstrated that the fermentation of TCM formulas is a complex process.

Due to the lack of reference standards for quantitation and poor baseline separation, only ten representative compounds with high contents were subjected to targeted analysis to illustrate the effects of processing. 
For puerarin, daidzin, daidzein, liquiritin and liquiritigenin, the results of the targeted quantification were consistent with those obtained in the untargeted studies, thus demonstrating that the hydrolysis of $O$-glycosides occurred due to the effect of $\beta$-glucosidase of SC $[2,41,42]$ and further supporting speculation that $\mathrm{C}$-glucoside is more difficult to transform via biotransformation with SC. In addition, the variation trends of coptisine, berberine, palmatine and baicalin in the targeted quantification corresponded with the results of the untargeted metabolomics, suggesting that multiple reactions might simultaneously occur, resulting in a dynamic equilibrium (Figs. 5, 6). Interestingly, the increasing trend of baicalein in the targeted analysis was highly different from the significant increase in baicalein observed in the untargeted analysis. Thus, we conclude that baicalein is altered slightly due to the dynamic equilibrium between flavone $O$-glucuronides and their derivatives. According to these results, our integrated strategy was useful for screening, matching and identifying the metabolites of FGQD.

Increasing evidence has indicated that the ten targeted compounds detected in raw and fermented GQD have various regulatory actions against T2DM. The antidiabetic effects of Gegen isoflavones have been demonstrated in several studies [43-46]. A previous study showed that both puerarin and daidzein from Gegen could reduce FBG and improve ISI and hyperlipidaemia in diabetic mice or rats [43-45], whereas daidzin showed an opposite effect by stimulating glucose uptake [46]. In addition, it was reported that daidzein can improve plasma TC, TG and HDL-C concentrations in $d b / d b$ mice [43]. Gaur reported that liquiritigenin from Gancao could be used as a possible lead for the control of FBG levels [47]. Several studies have shown that daidzein and liquiritigenin, which are small, hydrophobic molecules, are absorbed faster and in higher amounts than their glucosides, daidzin and liquiritin, in humans [44]. Thus, the increasing trends of flavone aglycones (daidzein and liquiritigenin) and isoflavone $C$-glycosides (puerarin), as well as other homologous compounds, might be helpful for explaining the greater anti-diabetic effects of FGQD, which occur partially via regulation of the levels of ISI, TC, TG, and HDL. Moreover, baicalin and baicalein from Huangqin have been demonstrated to exhibit excellent anti-diabetic activities [48-50]. Berberine, palmatine and coptisine have also been reported to exert antidiabetic effects involved in improving insulin resistance and secretion and promoting glucose consumption in 3T3L1 murine pre-adipocytes cells [51-53]. Thus, the stable contents of baicalin, baicalein, coptisine, berberine and palmatine, which showed obvious antidiabetic effects, as well as other compounds in FGQD, may contribute to the observed anti-diabetic effects. Taken together, these findings will help enhance our understanding of the greater anti-diabetic effects of FGQD.

\section{Conclusions}

In the present study, the antidiabetic effects and chemical profiles between GQD and FGQD were systematically compared. The anti-diabetic effects of FGQD were more potent than those of GQD, suggesting that the anti-diabetic activities of TCM formulas might be improved by applying fermentation technology. Moreover, the integration of chromatographic technique-based untargeted metabolomics and targeted analysis can be considered a useful approach for systematically exploring the chemical profiles of raw and fermented formulas. The increasing activities might be ascribed to the main constituents of transformation between GQD and FGQD. To ensure the therapeutic effects and safety of FGQD, the role of fermentation in processing should be further studied.

\section{Additional files}

Additional file 1. Minimum standards of reporting checklist.

Additional file 2: Table S1. Calibration curves, LODs, LOQs, repeatability, accuracy and stability of the quantitative assays for 10 analytes in GQD. Figure S1. Workflow of the untargeted metabolomic analysis. Figure S2 Effects of HM, GQD and FGQD on the FBG levels in T2DM rats. ${ }^{* *} p<0.01$ DM vs NC; $p<0.05, \# p<0.01$ HM vs DM; $p<0.05$, 蜘 $p<0.01$ DM vs GQD; $\triangle_{p<0.05,} \triangle \triangle_{p}<0.05$ FGQD vs DM. Figure S3. Chemical structures of the compounds identified in GQD. P: Pueraria Lobatae Radix; S: Scutellariae Radix; C: Coptidis Rhizoma; G: Glycyrrhizae Radix et Rhizoma Praeparata cum Melle. Figure S4. Extracted ion chromatograms of 133 constituents from GQD. P: Pueraria Lobatae Radix; S: Scutellariae Radix; C: Coptidis Rhizoma; G: Glycyrrhizae Radix et Rhizoma Praeparata cum Melle. Figure S5. PCA score plots of GQD and FGQD. A: negative ion; B: positive ion. Figure S6. Representative HPLC chromatograms of ten marker compounds at $254 \mathrm{~nm}$ and $276 \mathrm{~nm}$. P11: puerarin, P18: daidzin, P35: daidzein, C14: coptisine, C18: palmatine, C19: berberine, G3: liquiritin, G12: liquiritigenin, S19: baicalin, S37: baicalein.

\section{Abbreviations}

GQD: Ge-Gen-Qin-Lian decoction; FGQD: fermented Ge-Gen-Qin-Lian decoction; TCM: traditional Chinese medicine; SC: Saccharomyces cerevisiae; HPLC: high-performance liquid chromatography; MS: mass spectrometry; PD: potato dextrose; T2DM: type 2 diabetes mellitus; STZ: streptozotocin; NC: control group; HFD: high-fat diet; FBG: fasting blood glucose; HM: metformin hydrochloride; TC: total serum cholesterol; TG: triglycerides; HDL-C: high-density lipoprotein cholesterol; LDL-C: low-density lipoprotein cholesterol; FINS: fast serum insulin; HOMA-IR: homeostasis model assessment-insulin resistance; RDA: Retro-Diels-Alder; QCs: quality control samples; PCA: principal component analysis; OPLS-DA: orthogonal projection to latent structure-discriminant analysis; AGC: automatic gain control; NCE: normalized collision energies; EIC: extracted ion chromatography.

\section{Authors' contributions}

$Y Y, C D, X Q$, and $Q S$ conceived and designed the experiments. MZ, JL and JJ performed the experiments. YY wrote the manuscript. ZL and AL revised the manuscript. All authors read and approved the final manuscript. 


\begin{abstract}
Author details
${ }_{1}^{1}$ Modern Research Center for Traditional Chinese Medicine of Shanxi University, No. 92, Wucheng Road, Taiyuan 030006, Shanxi, China. ${ }^{2}$ School of Traditional Chinese Materia Medica, Shanxi University of Chinese Medicine, No. 121, Daxue Street, Taiyuan 030619, Shanxi, China. ${ }^{3}$ College of Chemistry and Chemical Engineering of Shanxi University, No. 92, Wucheng Road, Taiyuan 030006, Shanxi, China.
\end{abstract}

\section{Acknowledgements}

We thank the Scientific Instrument Center of Shanxi University for Q Exactive HR-MS analysis.

\section{Competing interests}

The authors declare that they have no competing interests.

\section{Availability of data and materials}

All data generated or analysed during this study are included in this published article.

\section{Consent for publication}

All authors consent to publication of this study in the journal Chinese Medicine.

\section{Ethics approval and consent to participate}

The animal study was performed according to the International Rules Concerning Animal Experiments and the Internationally Accepted Ethical Principles for Laboratory Animal Use and Care.

\section{Funding}

This research was supported by the National Natural Science Foundation of China (No. 81273659).

\section{Publisher's Note}

Springer Nature remains neutral with regard to jurisdictional claims in published maps and institutional affiliations.

\section{Received: 25 July 2018 Accepted: 24 September 2018}

Published online: 26 October 2018

\section{References}

1. Dong JW, Cai L, Xiong J, Chen XH, Wang WY, Shen N, Liu BL, Ding ZT. Improving the antioxidant and antibacterial activities of fermented Bletilla striata with Fusarium avenaceum and Fusarium oxysporum. Process Biochem. 2015;50(1):8-13.

2. Huang $Q$, Zhang $H$, Xue D. Enhancement of antioxidant activity of Radix Puerariae and red yeast rice by mixed fermentation with Monascus purpureus. Food Chem. 2017;226:89-94.

3. Fu Y, Yin Z, Wu L, Yin C. Fermentation of ginseng extracts by Penicillium simplicissimum GS33 and antiovarian cancer activity of fermented products. World J Microbiol Biotechnol. 2014;30(3):1019.

4. Tan L, Zhang X, Mei Z, Wang J, Li X, Huang W, Yang S. Fermented Chinese formula Shuan-Tong-Ling protects brain microvascular endothelial cells against oxidative stress injury. Evid Based Complement Altern Med. 2016:2016:5154290.

5. Kim DS, Um YR, Ma JY. Flavonoid content, free radical scavenging and increase in xanthine oxidase inhibitory activity in Galgeun-tang following fermentation with Lactobacillus plantarum. Mol Med Rep. 2014;10(5):2689-93.

6. Huang Q, Zhang H, Xue D. Enhancement of antioxidant activity of Radix Puerariae and red yeast rice by mixed fermentation with Monascus purpureus. Food Chem. 2017;226:89-94.

7. Fu Y, Yin Z, Wu L, Yin C. Fermentation of ginseng extracts by Penicillium simplicissimum GS33 and anti-ovarian cancer activity of fermented products. World J Microbiol Biotechnol. 2014;30(3):1019.

8. Kim DS, Um YR, Ma JY. Flavonoid content, free radical scavenging and increase in xanthine oxidase inhibitory activity in Galgeun-tang following fermentation with Lactobacillus plantarum. Mol Med Rep. 2014;10(5):2689.
9. Tan L, Zhang X, Mei Z, Wang J, Li X, Huang W, Yang S. Fermented chinese formula Shuan-Tong-Ling protects brain microvascular endothelial cells against oxidative stress injury. Evid Based Complement Altern Med. 2016;2016:5154290

10. Limón Rl, Peñas E, Torino Ml, Martínez-Villaluenga C, Dueñas M, Frias J. Fermentation enhances the content of bioactive compounds in kidney bean extracts. Food Chem. 2015;172:343-52

11. Li ZY, He P, Sun HF, Qin XM, Du GH. ${ }^{1} H$ NMR based metabolomic study of antifatigue effect of Astragali Radix. Euro J Integr Med. 2014:6(6):3022-30.

12. Zhou H, Liang J, Lv D, Hu Y, Zhu Y, Si J, Wu S. Characterization of phenolics of Sarcandra glabra by non-targeted high-performance liquid chromatography fingerprinting and following targeted electrospray ionisation tandem mass spectrometry/time-of-flight mass spectrometry analyses. Food Chem. 2013;138(4):2390-8.

13. Chen Y, Zhou Z, Yang W, Bi N, Xu J, He J, Zhang R, Wang L, Abliz Z. Development of a data-independent targeted metabolomics method for relative quantification using liquid chromatography coupled with tandem mass spectrometry. Anal Chem. 2017;89:6954-62.

14. Naz S, Vallejo M, García A, Barbas C. Method validation strategies involved in non-targeted metabolomics. J Chromatogr A. 2014;1353:99-105.

15. Li Z, Xu JD, Zhou SS, Qian M, Ming K, Hong S, Li XY, Duan SM, Xu J, Li SL. Integrating targeted glycomics and untargeted metabolomics to investigate the processing chemistry of herbal medicines, a case study on Rehmanniae Radix. J Chromatogr A. 2016;1472:74-87.

16. Cao G, Zhang C, Zhang Y, Cong X, Cai H, Cai B, Li X, Yao J. Global detection and identification of components from crude and processed traditional Chinese medicine by liquid chromatography connected with hybrid ion trap and time-of-flight-mass spectrometry. J Sep Sci. 2011;34(15):1845-52.

17. Qi W, Wei S, Xue Q, Shuai J, Yi K, Zhang ZX, Tao B, Guo DA, Ye M. Simultaneous quantification of 50 bioactive compounds of the traditional Chinese medicine formula Gegen-Qinlian decoction using ultra-high performance liquid chromatography coupled with tandem mass spectrometry. J Chromatogr A. 2016;1454:15-25.

18. Miao WJ, Wang Q, Bo T, Ye M, Qiao X, Yang WZ, Xiang C, Guan XY, Guo DA. Rapid characterization of chemical constituents and rats metabolites of the traditional Chinese patent medicine Gegen-Qinlian-Wan by UHPLC/ DAD/qTOF-MS. J Pharm Biomed Anal. 2013;72:99-108. https://doi. org/10.1016/j.jpba.2012.09.015.

19. Zhang $\mathrm{CH}$, Xu GL, Liu YH, Rao Y, Yu RY, Zhang ZW, Wang YS, Tao L. Antidiabetic activities of Gegen Qinlian decoction in high-fat diet combined with streptozotocin-induced diabetic rats and in 3T3-L1 adipocytes. Phytomedicine. 2013;20(3-4):221-9.

20. Tong XL, Zhao LH, Lian FM, Qiang Z, Le X, Zhang J, Chen XY, Ji HY. Clinical observations on the dose-effect relationship of Gegen Qin Lian decoction on 54 out-patients with type 2 diabetes. J Tradit Chin Med. 2011;31(1):56-9.

21. Tian N, Wang J, Wang P, Song X, Yang M, Kong L. NMR-based metabonomic study of Chinese medicine Gegen Qinlian decoction as an effective treatment for type 2 diabetes in rats. Metabolomics. 2013;9(6):1228-42.

22. Chen H, Guo J, Pang B, Zhao L, Tong X. Application of herbal medicines with bitter flavor and cold property on treating diabetes mellitus. Evid Based Complement Altern Med. 2015;2015:529491.

23. Li H, Zhao L, Zhang B, Jiang Y, Wang X, Guo Y, Liu H, Li S, Tong X. A network pharmacology approach to determine active compounds and action mechanisms of ge-gen-qin-lian decoction for treatment of type 2 diabetes. Evid Based Complement Altern Med. 2014;2014:495840. https:// doi.org/10.1155/2014/495840.

24. Jiang S, Du P, An L, Yuan G, Sun Z. Anti-diabetic effect of Coptis chinensis polysaccharide in high-fat diet with STZ-induced diabetic mice. Int J Biol Macromol. 2013:55(3):118-22.

25. Ji YJ, Lim Y, Min SM, Ji YK, Kwon O. Onion peel extracts ameliorate hyperglycemia and insulin resistance in high fat diet/streptozotocin-induced diabetic rats. Nutr Metab. 2011;8(1):18.

26. Du G, Zhao H, Zhang Q, Li GH, Yang F, Wang Y, Li Y, Wang Y. A rapid method for simultaneous determination of 14 phenolic compounds in Radix Puerariae using microwave-assisted extraction and ultra high performance liquid chromatography coupled with diode array detection and time-of-flight mass spectrometry. J Chromatogr A. 2010;1217(5):705-14. 
27. Xue Q, Qi W, Wei S, Yi Q, Yao X, Rong A, Guo DA, Ye M. A chemical profiling solution for Chinese medicine formulas using comprehensive and loopbased multiple heart-cutting two-dimensional liquid chromatography coupled with quadrupole time-of-flight mass spectrometry. J Chromatogr A. 2016;1438:198-204.

28. Yang ZW, Xu F, Liu X, Cao Y, Tang Q, Chen QY, Shang MY, Liu GX, Wang X, Cai SQ. An untargeted metabolomics approach to determine component differences and variation in their in vivo distribution between Kuqin and Ziqin, two commercial specifications of Scutellaria Radix. RSC Adv. 2017;7(86):54682-95.

29. Zhang L, Zhang RW, Li Q, Lian JW, Liang J, Chen XH, Bi KS. Development of the fingerprints for the quality evaluation of Scutellariae Radix by HPLC-DAD and LC-MS-MS. Chromatographia. 2007;66(1):13-20.

30. Islam MN, Downey F, Ng KY. Comprehensive profiling of flavonoids in Scutellaria incana L. using LC-Q-TOF-MS. Acta Chromatogr. 2013;25(3):555-69.

31. Han J, Ye M, Xu M, Sun J, Wang B, Guo DA. Characterization of flavonoids in the traditional Chinese herbal medicine-Huangqin by liquid chromatography coupled with electrospray ionization mass spectrometry. J Chromatogr B Analyt Technol Biomed Life Sci. 2007;848(2):355-62.

32. Montoro P, Maldini M, Russo M, Postorino S, Piacente S, Pizza C. Metabolic profiling of roots of liquorice (Glycyrrhiza glabra) from different geographical areas by ESI/MS/MS and determination of major metabolites by LC-ESI/MS and LC-ESI/MS/MS. J Pharm Biomed Anal. 2011;54(3):535-44.

33. Xue Q, Ru L, Wei S, Miao WJ, Jia L, Chen HB, Guo DA, Ye M. A targeted strategy to analyze untargeted mass spectral data: rapid chemical profiling of Scutellaria baicalensis using ultra-high performance liquid chromatography coupled with hybrid quadrupole orbitrap mass spectrometry and key ion filtering. J Chromatogr A. 2016;1441:83-95.

34. Qiao X, Song W, Ji S, Wang Q, Guo DA, Ye M. Separation and characterization of phenolic compounds and triterpenoid saponins in licorice (Glycyrrhiza uralensis) using mobile phase-dependent reversed-phase $x$ reversed-phase comprehensive two-dimensional liquid chromatography coupled with mass spectrometry. J Chromatogr A. 2015;1402:36-45.

35. Wang D, Liu Z, Guo M, Liu S. Structural elucidation and identification of alkaloids in Rhizoma Coptidis by electrospray ionization tandem mass spectrometry. J Mass Spectrom. 2004;39(11):1356-65.

36. Chuang WC, Young DS, Liu LK, Sheu SJ. Liquid chromatographic-electrospray mass spectrometric analysis of Coptidis Rhizoma. J Chromatogr A. 1996;755(1):19-26.

37. Hirakura K, Morita M, Nakajima K, Sugama K, Takagi K, Niitsu K, Ikeya Y, Maruno M, Okada M. Phenolic glucosides from the root of Pueraria lobata. Phytochemistry. 1997;46(5):921-8.

38. Xue Q, Qi W, Shuang W, Miao W, Li Y, Cheng X, Guo DA, Ye M. Compound to extract to formulation: a knowledge-transmitting approach for metabolites identification of Gegen-Qinlian decoction, a traditional Chinese medicine formula. Sci Rep. 2016;6:39534.

39. Choi RJ, Ha IJ, Choi JS, Park YM, Kim YS. Biotransformation of flavonoid-7-O-glucuronides by $\beta$-glucuronidases. Nat Prod Sci. 2010; 16(1):1-5. http://www.koreascience.or.kr/article/ArticleFullRecord.jsp?cn=E1HSB Y_2010_v16n1_1. Accepted 3 Mar 2010.
40. Wang C, Yin YH, Wei YJ, Shi ZQ, Liu JQ, Liu LF, Xin GZ. Rapid identification of herbal compounds derived metabolites using Zebrafish larvae as the biotransformation system. J Chromatogr A. 2017;1515:100-8.

41. Hernández LF, Espinosa JC, Fernández-González M, Briones A. $\beta$-Glucosidase activity in a Saccharomyces cerevisiae wine strain. Int J Food Microbiol. 2003;80(2):171-6.

42. Amor IL, Hehn A, Guedone E, Ghedira K, Engasser JM, Chekir-Ghedrira L, Ghoul M. Biotransformation of naringenin to eriodictyol by Saccharomyces cerevisiea functionally expressing flavonoid $3^{\prime}$ hydroxylase. Nat Prod Commun. 2010;5(12):1893-8.

43. Ae PS, Choi MS, Cho SY, Seo JS, Jung UJ, Kim MJ, Sung MK, Park YB, Lee MK. Genistein and daidzein modulate hepatic glucose and lipid regulating enzyme activities in C57BL/KsJ-db/db mice. Life Sci. 2006;79(12):1207-13.

44. Kwon DY, Daily JW III, Kim HJ, Park S. Antidiabetic effects of fermented soybean products on type 2 diabetes. Nutr Res. 2010;30(1):1-13.

45. Wu K, Liang T, Duan X, Xu L, Zhang K, Li R. Anti-diabetic effects of puerarin, isolated from Pueraria lobata (Willd), on streptozotocin-diabetogenic mice through promoting insulin expression and ameliorating metabolic function. Food Chem Toxicol. 2013;60(10):341-7.

46. Meezan E, Meezan EM, Jones K, Moore R, Barnes S, Prasain JK. Contrasting effects of puerarin and daidzin on glucose homeostasis in mice. J Agric Food Chem. 2005;53(22):8760-7.

47. Gaur R, Yadav KS, Verma RK, Yadav NP, Bhakuni RS. In vivo anti-diabetic activity of derivatives of isoliquiritigenin and liquiritigenin. Phytomedicine. 2014;21(4):415-22.

48. Hsu FL, Liu IM, Kuo DH, Chen WC, Su HC, Cheng JT. Antihyperglycemic effect of puerarin in streptozotocin-induced diabetic rats. J Nat Prod. 2003;66(6):788-92.

49. Yu F, Luo J, Jia Z, Wei Z, Zhou K, Gilbert E, Liu D. Baicalein protects against type 2 diabetes via promoting islet $\beta$-cell function in obese diabetic mice. Int J Endocrinol. 2014;2014:846742. https://doi.org/10.1155/2014/846742.

50. Ahad A, Mujeeb M, Ahsan H, Siddiqui WA. Prophylactic effect of baicalein against renal dysfunction in type 2 diabetic rats. Biochimie. 2014;106:101-10.

51. Dong Y, Chen YT, Yang YX, Zhou XJ, Dai SJ, Tong JF, Shou D, Li C. Metabolomics study of type 2 diabetes mellitus and the antidiabetic effect of berberine in zucker diabetic fatty rats using UPLC-ESI-HDMS. Phytother Res. 2016;30(5):823-8.

52. Aggarwal BB, Prasad S, Reuter S, Kannappan R, Yadev VR, Park B, Kim JH, Gupta SC, Phromnoi K, Sundaram C, Prasad S, Chaturvedi MM, Sung B. Identification of novel anti-inflammatory agents from ayurvedic medicine for prevention of chronic diseases: "reverse pharmacology" and "bedside to bench" approach. Curr Drug Targets. 2011;12(11):1595-653.

53. Jung HA, Na YY, Bae HJ, Min BS, Choi JS. Inhibitory activities of the alkaloids from Coptidis Rhizoma against aldose reductase. Arch Pharm Res. 2008;31(11):1405-12.

\footnotetext{
Ready to submit your research? Choose BMC and benefit from:

- fast, convenient online submission

- thorough peer review by experienced researchers in your field

- rapid publication on acceptance

- support for research data, including large and complex data types

- gold Open Access which fosters wider collaboration and increased citations

- maximum visibility for your research: over 100M website views per year
}

At $\mathrm{BMC}$, research is always in progress.

Learn more biomedcentral.com/submissions 